\title{
Nicotine Stimulates Peristalsis in N. Vectensis: From Behavior to Nicotinic Receptor Genes
}

Joanne Figueiredo ( $\sigma$ jfigueiredo@smithtown.k12.ny.us )

Smithtown High School West, United States

Joshua Rest

Stony Brook University

Sarah Adamo

Northeastern University

Rebecca Grella

Brentwood High School, NY

James Dilger

Stony Brook University

Research Article

Keywords: nAChRs, acetylcholine, Nicotinic

Posted Date: February 16th, 2021

DOI: https://doi.org/10.21203/rs.3.rs-195699/v1

License: (c) (i) This work is licensed under a Creative Commons Attribution 4.0 International License. Read Full License 


\section{Abstract}

Nicotinic acetylcholine receptors (nAChRs) play a pivotal role in the nervous system, and there is great interest in understanding their evolution. Cnidarians, an ancestral group of metazoans, display an early nervous system utilizing complex neural architecture that provides insight into the early evolution and function of nAChRs. Using the anthozoan Nematostella vectensis, we found that the rate of peristalsis, a radial constriction of the body column, is modulated by nicotinic agonists. Nicotine caused a robust, dose dependent increase in the rate of peristalsis. The peristalsis rate also increased in response to ACh in the presence of edrophonium, an acetylcholine esterase inhibitor. Thus, $N$. vectensis possesses functional nAChRs. We developed a phylogeny of nAChRs from 15 cnidarian and 16 bilaterian species. Our results indicate that the large number of cnidarian and bilaterian nAChR gene copies is convergent, resulting from gene expansions after the two lineages diverged. These independent expansions led to an average of 31 and 34 paralogous nAChR gene copies in sampled bilaterians and cnidarians, respectively. The $N$. vectensis proteome contains 49 paralogous $\mathrm{nAChR}$ copies. The independent expansions indicate that $\mathrm{nAChRs}$ receptors in cnidarians may have distinct pharmacological and biochemical characteristics in comparison to their bilaterian counterparts.

\section{Introduction}

The phylum Cnidaria is made up of a diverse group of organisms including jellyfish, corals, hydroids and sea anemones. A recent phylogenetic analysis of all metazoan phyla places Cnidaria as sister to Bilateria ${ }^{1}$. An important feature in these organisms is the presence of a well-defined nervous system: nerve nets in cnidarians and a central nervous system in bilaterians. On a molecular level, both groups possess voltage-gated and ligand-gated ion channels which suggests similar neuronal signaling processes ${ }^{2}$. Thus, cnidarians have the potential to provide insight into the evolution of the nervous system.

Elucidation of which neurochemical signals cnidarians utilize has been hampered by the paucity of clear physiologic and behavioral responses to these molecules. Species for which meaningful behavioral assays exist include luminescence in Renilla kollikeri ${ }^{3,4}$, photic behavior in Tripedalia cystophora ${ }^{5}$ and tentacle contraction in Hydra vulgaris ${ }^{6}$ and Nematostella vectensis ${ }^{7}$. A challenge in studying cnidarians has been the lack of representative species with fully sequenced genomes. Until 2019, only the genomes of $N$. vectensis ${ }^{8}$, Acropora digitifera ${ }^{9}, H$. vulgaris ${ }^{10}$, and Exaiptasia diaphana ${ }^{11}$, were available.

The sea anemone $N$. vectensis has great potential to unlock some of the secrets of cnidarian neurotransmission. This anthozoan cnidarian has emerged as an important model organism ${ }^{12}$. In addition to having a fully sequenced genome ${ }^{8}$, it is easy to cultivate and propagate. Furthermore, it has been shown to possess the complete repertoire of nervous system proteins associated with synaptic transmission in vertebrates ${ }^{13}$. The $N$. vectensis genome contains genes that encode receptors and enzymes related to classic neurotransmitters such as acetylcholine, glutamate, GABA, and ATP; proteins associated with biogenic amines and other non-peptidergic transmitters (such as DOPA and adrenaline); as well as sequences that code for proteins associated with neuropeptides (such as RFamide) ${ }^{13}$. Here, we focus on the nicotinic acetylcholine receptor (nAChR) ion channel protein.

nAChRs are members of the pentameric ligand-gated ion channel superfamily. These proteins possess an extracellular stretch of 13 amino acids flanked by a cysteine at each end (cys-loop), an extracellular ligand binding site, four transmembrane helices (TM1-TM4), and exhibit a pentameric quaternary structure with a central pore through which ions can pass ${ }^{14}$. Receptors are composed of five subunits, which may be encoded by one or multiple paralogous gene copies. Nicotinic receptors, along with 5HT3 and Zinc-activated channels form cationic pores, while $\mathrm{GABA}_{A}$ and glycine receptors form anionic channels. In the case of the $\mathrm{nAChR}$, the endogenous ligand is acetylcholine, but nicotine (from which the receptor gets its name) is also a potent ligand. In vertebrates, nAChRs participate in various functions including fast synaptic transmission at the neuromuscular junction and synapses in the central nervous system, cognitive and pathophysiological effects of nicotine addiction, learning and memory, and the immune system (reviewed in ${ }^{15}$.

nAChRs have been studied in other cnidarians where behavioral or physiologic assays exist. Cholinergic agonists induce muscle contractions and bioelectric activity in $\mathrm{H}$. vulgaris ${ }^{16}$ and the anemone Bunodosoma caissarum ${ }^{17}$. Biochemical studies have provided evidence that acetylcholinesterases are present in the anenome Actinia equina ${ }^{18}$. Recent evidence shows that both ACh 
and nicotine induce tentacle contractions in $N$. vectensis and that this effect can be blocked by nicotinic antagonists such as mecamylamine ${ }^{7}$.

The presence of genes that code for cholinergic system proteins in $N$. vectensis was first detailed by ${ }^{13}$. He described 20 paralogous cholinergic genes, including 12 nAChR genes, 3 choline acetyltransferase genes, and 5 acetylcholinesterase genes. Further molecular evidence for cholinergic function was provided by a study demonstrating that nAChRs are expressed during $N$. vectensis development ${ }^{19}$. This work presented evidence that these receptors can be found in the ectoderm and are enriched in the apical organ in planulae. A recent extension of this work showed that $N$. vectensis possesses $26 \mathrm{nAChR}$ gene paralogs and that the transcriptional products of these genes are expressed during development in neurons, muscles, and gonads ${ }^{7}$.

The goal of the work presented here was to explore both experimental and phylogenomic evidence for the functionality of nAChR receptors. We provide clear evidence $N$. vectensis has a robust physiologic response to nicotine and present a detailed phylogeny of the nAChR in cnidarians and bilaterians. Although a number of different behaviors have been identified in $N$. vectensis including feeding, swimming, sudden contraction ${ }^{20}$ and peristalsis ${ }^{21}$, only tentacle contraction has been shown to be under neuronal control ${ }^{7}$. Here we demonstrate that peristalsis, a periodic radial constriction of the body column progressing as a wave that begins approximately half-way down the body column and continues to the foot or physa, can be modulated by nicotinic cholinergic agonists. Specifically, we show that the rate of peristalsis is dramatically increased in a dose-dependent way by nicotine. Our results suggest that peristaltic waves are coupled to nicotinic cholinergic receptors in $N$. vectensis and that this behavior can be used to better characterize the function of these receptors. In addition, we examine the phylogenetic relationship of cnidarian nAChRs in comparison to other eumetazoan receptors, infer dramatic parallel evolution in gene copy number between the cnidarians and eumetazoans, and evaluate evidence for the closest related bilaterian receptors.

\section{Material And Methods}

\section{Animal Care}

Cultures of $N$. vectensis were maintained in $12 \mathrm{ppt}$ artificial sea water (ASW) at $18^{\circ} \mathrm{C}$ under artificial light $(12 \mathrm{~h}$ light:12 h dark). They were fed three times per week with freshly hatched Artemia salina nauplii and were cleaned after each feeding ${ }^{20,22}$. Sexual reproduction was induced by the method of ${ }^{23}$. Fertilized eggs were removed from adult cultures and placed in separate $39 \mathrm{~mm}$ culture dishes in $12 \mathrm{ppt}$ ASW. Eggs were incubated at $18^{\circ} \mathrm{C}$ for $6-8 \mathrm{~d}$ until the 4 tentacle juvenile polyps appeared. Polyps $8-21 \mathrm{~d}$ old were used in experiments on peristalsis. Developing polyps were not fed until day 21 post fertilization.

\section{Behavioral Experiments}

Polyps were placed in 6 well culture plates $24 \mathrm{~h}$ before experiments. Approximately $20-25$ polyps were added to $8 \mathrm{ml}$ of $12 \mathrm{ppt}$ ASW in each well. Animals were carefully transferred using a $12 \mathrm{~cm}$ glass pipette so that 8-12 animals were visible in the field of view on a Zeiss Stemi Stereo Microscope. Experiments were initiated by recording a 5 min video under control conditions. Next, the appropriate dose of chemical treatment was added to the well and a 5 min equilibration period was allowed. Finally, a second 5 min video was recorded with the animals still in their original relative positions. This before and after treatment design made it possible for each animal to act as its own control. The sequence of treatment conditions was varied randomly. In some experiments a second chemical treatment was added to the well. In these cases, a 5 min equilibration period preceded a 5 min video of the stimulated polyps.

Videos were recorded using an Excelis digital camera attached to the Zeiss microscope. Videos were converted to MP4 format using HandBrake for analysis. Control and treatment videos were opened side by side using two windows on the computer monitor to ensure the identity of control and stimulated animals. In order to count the number of waves, videos were watched at 10X speed. Waves that began before the $5 \mathrm{~min}$ recording session were counted but waves that did not complete before the end of the session were not. Animals were excluded from analysis if their position changed so that they were not in the field of view during both their control and treatment states. Each animal was used for only one experiment and only two wave frequency values are obtained from each animal, one for control and one for treatment. The speed of peristaltic waves in a small set of animals was determined by measuring the locations of the animal's mouth and the constricted region at $0.8 \mathrm{~s}$ intervals using the video 
analysis program Tracker v4.96 (http://physlets.org/tracker/, a project of Open Source Physics http://www.compadre.org/OSP/). A plot of the distance between the mouth and constriction vs time was fit by linear regression. The slope of the regression line provided the wave speed.

\section{Statistical Analysis}

Histograms of the number of peristaltic waves during a 5 min interval do not exhibit a normal distribution. Because each animal served as its own control, we used the nonparametric, Wilcoxon signed rank test, which is used for paired samples to test for differences in a repeated measures design. In cases where we compared waves under three conditions, the Friedman test (the nonparametric equivalent of ANOVA) was employed and was followed by Dunn's Multiple Comparison Test. Differences were considered significant when $\mathrm{p}<0.05$. Prism v6 (GraphPad Software, Inc., San Diego, CA, USA) was used for statistical tests and Igor Pro v8.04 (Wavemetrics, Inc., Oswego, OR, USA) was used for graphing of behavioral data. Violin plots were constructed as boxes showing the 25,50 and 75 percentiles, the feathers showing the full range of the data points and the solid shapes showing a smoothed representation of the distribution.

\section{Gene Family Identification, Alignment, and Phylogeny}

We created a seed alignment for studying two cationic pentameric ligand gated channels by selecting 14 well-characterized human nAChR proteins (each encoded by a distinct gene) and two Drosophila melanogasternAChR proteins, as well as 3 human $5 \mathrm{HT} 3$ receptor proteins (Supplementary Table S1). We aligned these using t_coffee $\mathrm{v} 12^{24}$ in accurate mode, and then trimmed the terminal ends to retain conserved regions, including loop A, the cys-loop, loop B, loop C, and transmembrane domains 1-3 (but excluding the poorly conserved transmembrane domain 4). We built a hidden Markov model (HMM) using this seed alignment using hmmbuild in hmmer v3.1b2 ${ }^{25}$.

For a query database, we examined 15 cnidarian predicted proteomes, in addition to selecting representatives from available xenocoelemorph, protostome, echinoderm, and hemichordate proteomes (31 proteomes; Supplementary Table S2). When available, we used proteomes with only a single isoform per gene, as curated by uniprot ${ }^{26}$. For all Genbank proteomes, we used SeqKit $0.12 .1^{27}$ to retain the longest isoform per gene. We only included proteomes with at least $80 \%{ }^{28}$ completeness according to the eukaryota_odb9 reference set in BUSCO v3.0.2 ${ }^{28}$. For reference, we constructed a species tree based on relationships reported in the literature ${ }^{29-32}$, although some of these relationships, such as the position of Xenacoelomorpha, are unsettled.

We queried these proteomes using hmmsearch from hmmer and kept only the top scoring domain if there were multiple hits. We only retained proteins with an independent e-value less than $1 \times 10^{-50}$, and where the full protein length is at least 400 amino acids. We also removed any protein that did not have a cysteine at both the beginning and end of the cys-loop (positions 119 and 133 in our HMM) according to the hmmer alignment. We aligned proteins that passed these filters with muscle ${ }^{33}$, and then used the seq_reformat function in t_coffee to remove any sites with greater than $50 \%$ gaps.

We used IQ-TREE v.2.0-rc $1^{34}$ to produce a phylogeny based on this alignment using the WAG substitution matrix ${ }^{35}$ and FreeRate model of substitution heterogeneity ${ }^{36}$ with 10 categories. To avoid local minima, we chose the optimal tree from six independent runs, including four optimized for short input sequences with a smaller than default perturbation strength (0.2) and larger number (500) of stop iterations. Node support was estimated using the ultrafast bootstrap ${ }^{37}$ and SH-aLRT test ${ }^{38}$ and 10,000 replicates.

To assess alternative topologies, we swapped fully-resolved branches on the optimal maximum likelihood tree in TreeGraph ${ }^{39}$ and performed AU ${ }^{40}$ and ELW ${ }^{41}$ tests with 10,000 replicates in IQ-TREE. We identified a-like proteins by the presence of doublecysteine at positions 182-183 of the HMM based alignment. We reconstructed the ML ancestral states of a or non-a status using the fastAnc function in phytools ${ }^{42}$. The species and gene trees were visualized with ggtree ${ }^{43}$.

\section{Results And Discussion}

Contractions in 12ppt ASW. N. vectensis polyps occasionally undergo a slow peristaltic contraction that starts about halfway down the body column and ends at the foot or physa (Fig. 1a; Supplementary Video 1). The waves travel at $9.7 \pm 2.0 \mu \mathrm{m} / \mathrm{s}(6$ animals, 17 measurements) (Fig. 1b). In no case did we observe a second wave begin in an animal before the first wave had ended. Occasionally, we observed a wave traveling in the reverse direction (physa to mid-column). 
Polyps underwent an average of 2.5 peristaltic waves per 5 min interval (pooled measurements from 808 animals that were subsequently used in experiments with different treatments). However, the number of waves varied from zero to nine with a median value of 2 and was not normally distributed (Fig. 2a). We fitted the distribution to the sum of two weighted Poisson distributions. The means and $95 \%$ confidence limits of the fit were $0.47 \pm 0.11$ waves per $5 \mathrm{~min}(0.41 \pm 0.05$ weight $)$ and $3.94 \pm$ 0.34 waves per $5 \mathrm{~min}$. This suggests that $N$. vectensis polyps normally have two activity levels: low and high, with slightly more than half of the polyps being in the high activity group.

Wave frequency is increased by nicotine. The addition of nicotine (final concentration $185 \mu \mathrm{M}$ ) to the solution bathing the polyps dramatically increased the number of waves to an average of 6.0 waves per 5 min (median of 6 waves per 5 min, 187 animals, Supplementary Video 2). We fitted the distribution as the sum of two Poisson distributions but the fitted weight of one component was zero; the remaining Poisson component had a mean of $6.5 \pm 0.83$ waves per 5 min (Fig. 2b). Thus, all of the polyps contracted at an activity level higher than that of the high activity in the control. The speed of the contractions in the presence of $185 \mu \mathrm{M}$ nicotine was twice as fast as those under control conditions (Fig. 2c; $19.3 \pm 3.7 \mu \mathrm{m} / \mathrm{s}, 5$ animals, 21 measurements, $p<0.001$, ttest, the same set of animals as were used to obtain the control speed, Supplementary Figure S1).

The excitation induced when nicotine is added to the well is not due to the animals being disturbed by the addition of fluid. Figure 3 shows the results for a set of 56 animals recorded under control conditions and then again five minutes after the addition of $0.10 \mathrm{ml}$ of $12 \mathrm{ppt}$ ASW. There was no significant change in the distribution of waves. These same animals responded to the subsequent addition of nicotine to the well (final concentration $370 \mu \mathrm{M}$ ).

The stimulating effect of nicotine was dose dependent (Fig. 4). For three concentrations of nicotine, $93 \mu \mathrm{M}$ ( 63 animals), $185 \mu \mathrm{M}$ (169 animals) and $370 \mu \mathrm{M}$ (55 animals) and the corresponding controls, the mean frequency of waves increased by factors of 1.8 , 2.4 and 4.3, respectively. Linear regression of the waves vs. nicotine concentration data yielded a slope of $0.015 \pm 0.002$ waves/ $\mu \mathrm{M}(95 \%$ confidence limits) indicating that the dose-dependence is highly significant (Supplementary Figure S2).

Contractions in the presence of acetylcholine (ACh). Surprisingly, ACh did not stimulate peristalsis. At concentrations ranging from $10 \mu \mathrm{M}$ to $5 \mathrm{mM}$, ACh appeared to inhibit peristalsis, but the results were inconsistent. In the example shown in Fig. $5 \mathrm{a}, 500 \mu \mathrm{M}$ ACh had no effect on the frequency of waves. One possible explanation for the lack of a stimulatory effect of ACh is that acetylcholinesterases in $N$. vectensis may rapidly hydrolyze exogenously applied ACh. We tested this idea by first exposing polyps to the acetylcholinesterase inhibitor, edrophonium and then added ACh. $500 \mu \mathrm{M}$ edrophonium increased the frequency of waves from 1.50 per 5 min to 3.32 per 5 min (Fig. $5 b$ ). Subsequent addition of $500 \mu \mathrm{M}$ ACh increased the frequency of waves even further to 5.21 per $5 \mathrm{~min}$. This suggests that inhibition of acetylcholinesterase by $500 \mu \mathrm{M}$ edrophonium increases the effectiveness of both endogenous and exogenously applied ACh.

N. vectensiscontains a large number of nAChR paralogs. We hypothesized that our behavioral observation of dramatic, dosedependent peristalsis in response to nicotine is due to the presence of nAChRs. To test this hypothesis, we first identified all potential nAChR paralogs in the $N$. vectensis genome. To determine the extent that the details of this type of neurochemical signaling may be homologous or convergent with bilaterian systems, we determined the phylogenetic relationship of the $N$. vectensis $\mathrm{nAChRs}$ to other characterized $\mathrm{nAChR}$ genes. According to our conservative estimate, the $N$. vectensis proteome contains 49 nAChR copies (Supplementary Table S3), the most of any cnidarian sampled to date. All of these encoded proteins have the characteristic structural features found in mammalian nAChRs (Supplementary Figure S3) and many of the amino acid residues within the agonist/antagonist binding loops are identical to those found in human nAChR proteins (Supplementary Table S4). These paralogous proteins are encoded on 27 different scaffolds. Queries into previous expression sets indicate that all 49 genes examined are expressed with varying temporal dynamics and magnitude ${ }^{44-46}$. The 15 species of cnidarians we analyzed average 34 nAChR paralogs, ranging from 13 in H. vulgaris to 46 in Pocillopora damicornis (Supplementary Table S2). The sampled bilaterian proteomes contain, on average, $31 \mathrm{nAChR}$ paralogs (including Zinc/5HT3 receptors), ranging from 7 in Ciona intestinalis (ascidian) to 68 in Mizuhopecten yessoensis (scallop). Humans have 16 nAChR paralogs. We verified that the number of predicted nAChR genes in a genome is not strongly predicted by the completeness of the genome annotation (Supplementary Figure S4). In total, our final protein alignment includes 502 cnidarian genes and 498 bilaterian genes and contains 435 positions (Supplementary Table S5). 
A single lineage of nAChR genes underwent a dramatic expansion in Cnidaria. Our phylogeny is rooted with a zinc/5HT3 outgroup, the paralogous sister group to all other cationic subunits ("Gene Tree" in Fig. 6; Supplementary Figure S5). In this tree, the relationships among the bilaterian nAChRs generally agrees with typical relationships described in recent literature (e.g. $\left.{ }^{47}\right)$. Within this phylogeny, we observe the 502 cnidarian gene copies occur in two distinct clades. The first cnidarian clade contains genes present in a single copy in just five of the anthozoans (Fungia spp., Stylophora pistillata, Pocillopora damicornis, Acropora tenuis, and Porites asteroides). These five genes ("Cnidaria-2" in Fig. 6) are part of the divergent group (Branch E) related to Drosophila $\beta 3$ and found in xenoceolemorphs, protostomes, echinoderms, and hemichordates, but not in chordates.

The remaining 497 cnidarian genes (99\%) belong in a single large, cnidarian-specific clade; we call this clade the Cnidaria-group of nAChRs ("Cnidaria-group") (* in Fig. 6). All 49 N. vectensis nAChRs are found in the Cnidaria-group (purple polyps in Fig. 6). The Cnidaria-group is well supported as a clade ( $98.9 \%$ SH-aLRT, $100 \%$ UFboot support). The Cnidaria-group diverged after the a9/10 lineage and its relatives (branch D ; found in protostomes, echinoderms, hemichordates, and chordates). The Cnidaria-group is sister to a clade that includes the "B1-group" and the a7 group (branch A in Fig. 6) (67.2\% SH-aLRT, 60\% UFboot support). The B1group is a large clade of well-studied bilaterian nAChRs, including the $a 1-6, \beta 1-4, \delta, \varepsilon$, and $y$ subunits (branch $B$; found in all bilaterians studied, including the xenoceolemorph). The a7 group is found in protostomes, echinoderms, hemichordates, and chordates (branch C).

We tested our confidence in the placement of the Cnidaria-group as sister (closest relatives) to the B1 + a7 group using alternative topology tests (Supplementary Table S6), and were able to reject alternative placement (c-ELW and p-AU $<0.01$ for both; joining along branches $\mathrm{B}$ or $\mathrm{C}$ in Fig. 6). All other sister relationships to major groups we tested cannot be rejected by either test $(p>0.05)$. We cannot reject the Cnidaria-group as sister to the a9/10 group (branch D; found in protostomes, echinoderms, hemichordates, and chordates), the $D \beta 3 /$ divergent group ${ }^{48}$ (branch $\mathrm{E}$; found in protostomes, echinoderms, hemichordates, and some cnidarians; see below), B1 + a7 + a9/10 (branch F), or B1 + a7 + a9/10 +D 33 (branch G).

It appears that the 497 gene copies in the Cnidaria-group all expanded from a single common ancestral gene in ancient cnidarians. Gene duplications led to increases in nAChR copy number especially in anthozoans, including the Hexacorallia, and Scleractinia cndiarians. Intriguingly, this expansion in cnidarians tentatively appears to be the result of an expansion of the same stem lineage that also exhibited a large expansion in bilaterian lineages (B1-group; although we cannot reject some alternatives). This brings about several important questions. First, why was this specific lineage subject to dramatic expansion, independently but in parallel (convergently), in bilaterians and cnidarians? Second, in how many ways did the nAChR systems in bilaterians and cnidarians evolve in parallel? Here, we have shown that they evolved in parallel in terms of expansion of the gene family copy number, suggesting the convergent evolution of a complex nAChR system. However, do the same types of subunits exist? Are they assembled into homopentamers and heteropentamers of similar types to those of bilaterians? Are they equivalently tissue specific? And do the serve the same range of functions, or do they have new and marvelous functions to discover? Answers to these questions will require further phylogenetic and functional characterization.

The observation that various types of complexity has arisen in animals multiple times, and is not specific to bilaterians, is now becoming well-appreciated ${ }^{49}$. Indeed, the independent expansion of nAChR gene family copy number in bilaterians and cnidarians that we observe here is consistent with a similar inference for the entire ligand-gated, cys-loop receptor superfamily (which also includes $\mathrm{GABA}_{A}$ and Glycine anionic receptors; not considered here) in a study using two cnidarian genomes ${ }^{50}$. That study also inferred a similar pattern for glutamate-gated channels and voltage gated potassium channels, also suggesting repeated and independent occurrences of the expansion of nervous system complexity.

To consider the question of how early nAChRs evolved, we also searched in proteomes from earlier diverging metazoan lineages. We found no evidence of nAChRs in the poriferans Xestospongia testudinaria, Tethya wilhelma, Stylissa carteri, Ephydatia muelleri, Stylissa carteri, and Amphimedon queenslandica, or in the ctenophores Mnemiopsis leidyi and Pleurobrachia bachei (not shown). Accumulating phylogenomic evidence suggests that Bilateria and Cnidaria form a monophyletic group that does not include the Porifera and Ctenophora (e.g. ${ }^{51-53}$; although other work places Porifera as sister to cnidarians, as in ${ }^{54}$. The presence of nAChRs in Bilateria and Cnidaria supports their sister relationship and monophyly, with respect to poriferans and ctenophores; 
this distribution is the same as for the entire cys-loop receptor superfamily as well as acid sensing channel ${ }^{50}$, and has also been observed for other types as genes, such as for Hox/ParaHox, S50 and K50 PRD and HNF class homeodomains ${ }^{55}$.

Repeated evolution of non-asubunits. Of the 49 nAChR genes in N. vectensis, 44 encode a-like subunits ( $90 \%$ ), as defined by the presence of a double-cysteine in loop C (Supplementary Figure S3). The five genes encoding non-a subunits come from four clades that each evolved independently into the non-a state (Fig. 6, Supplementary Figure S5). Among all cnidarian nAChR genes, $83 \%(417 / 502)$ are a-like. In contrast, for bilaterians, only 57\% are a-like (256/453). In both cases, the non-a state has arisen independently numerous times from the ancestral, a-like nAChRs ancestor. Like $D \beta 3$, the Cnidaria-2 group is non-a.

There are no muscarinic cholinergic receptors in N. vectensis. To rule out the possibility that the behavioral, dose dependent peristalsis we observed is due to the presence of muscarinic cholinergic receptors (mAChRs), we searched for the closest related genes to mAChRs within $N$. vectensis and determined their phylogenetic placement among rhodopsin-like G-protein-coupled receptors. Previous work has shown that anthozoans like N. vectensis do not possess mAChRs (Anctil, 2009; Faltine-Gonzalez and Layden, 2019) whereas hydrozoans such as $H$. vulgaris do (Collins, 2013.) We therefore conducted a phylogenetic analysis of mAChRs and other related rhodopsin-like G-protein-coupled muscarinic receptors. We identified the 10 top matching genes to a mAChR-specific HMM from each $H$. vulgaris and $N$. vectensis (Supplementary Figure S6). We found genes from $H$. vulgaris are sister to the bilaterian mAChRs. In contrast, the $N$. vectensis genes are related to octopamine receptors, rather than mAChRs, consistent with a lack of mAChRs. We also compared these cnidarian sequences at the 14 amino acid sites known to be critical to ACh binding (Supplementary Table S7). We found that the $H$. vulgaris genes have an average of 8 identical amino acids (range 714) while $N$. vectensis genes have an average of only 3 critical amino acids (range 2-5). We therefore infer that the response we see in $N$. vectensis is mediated by nAChRs, rather than mAChRs.

\section{Summary}

We have studied the effect of cholinergic agonists on peristalsis in $N$. vectensis. In other cnidarians, peristalsis has been shown to play important physiologic roles such as fluid circulation ${ }^{56}$, digestion ${ }^{57}$ and locomotion ${ }^{58}$. We found that the rate of peristalsis in $N$. vectensis exhibited a dramatic and dose-dependent increase in response to nicotine. Additionally, in the presence of the acetylcholinesterase inhibitor, edrophonium, acetylcholine increased the rate of peristalsis. Our findings are largely in agreement with previous work that showed that nicotine and acetylcholine induce tentacle contraction and that nicotine induces radial contractions in $N$. vectensis polyps ${ }^{7}$. In that work, concentrations of nicotine from $2.5 \mathrm{mM}$ to $10 \mathrm{mM}$ were used. The behavioral method for assessing contractions described here is much more sensitive, showing a clear dose response from $90 \mu \mathrm{M}$ to $370 \mu \mathrm{M}$. These observations lead us to infer that peristalsis in $N$. vectensis is mediated by nicotinic cholinergic receptors.

We then focused on the relationship between cnidarian nAChR genes and the well-studied genes expressed by bilaterians. To do this, we identified nAChR copies encoded in the genomes of 16 bilaterians and 15 cnidarians. We found that bilaterians possess roughly equivalent numbers of paralogous $\mathrm{nAChR}$ gene copies, with $N$. vectensis encoding the highest number. A detailed gene tree suggests that the major nAChR cnidarian lineage came from a single stem lineage which has since undergone dramatic expansion. All 49 of the $N$. vectensis nAChR genes fall into this clade, which is sister to one of the stem bilaterian lineages. Independently, the bilaterian nAChR gene family also expanded to a high copy number from this and other stem lineages. Taken together, the work presented here shows that the high copy number of nAChR subunits in both Cnidaria and Bilateria is due to convergent and parallel expansion. In other words, there is no one-to-one relationship between the many subunits in bilaterians and the many subunits in cnidarians, since the cnidarian-group subunits all expanded from a single ancestral cnidarian copy. This convergent expansion is likely to have produced a set of cnidarian proteins that have distinct pharmacological and biochemical properties from those of their bilaterian counterparts.

\section{Declarations}

\section{Acknowledgements}

The authors thank Dr. Dianna K. Padilla and Dr. David Q. Matus for their insightful comments on early versions of the manuscript. 


\section{Author Contributions}

JCF, SA, RAG performed behavioral experiments. JSR performed genomic analysis. JPD provided statistical analysis of behavioral experiments and critical insight into building the HMM used to search proteomes for nicotinic receptors. JCF, JSR and JPD wrote the manuscript with input from SA and RAG.

\section{Competing Interests}

The authors declare no competing interests.

\section{References}

1. Laumer,C. E.et al.Revisitingmetazoanphylogenywithgenomicsamplingofallphyla.Proc. R. Soc. B Biol. Sci. (2019)doi:10.1098/rspb.2019.0831.

2. Sinigaglia, C., Busengdal, H., Lerner, A., Oliveri, P. \& Rentzsch, F. Molecular characterization of the apical organ of the anthozoan Nematostella vectensis.Dev. Biol.398,120-133(2015).

3. Hand, C. \& Uhlinger, K. R. The culture, sexual and asexual reproduction, and growth of the sea anemone Nematostella vectensis.Biol. Bull.182,169-176(1992).

4. Williams, R. B. Locomotory behaviour and functional morphology of Nematostella vectensis (Anthozoa: Actiniaria: Edwardsiidae): a contribution to a comparative study of burrowing behaviour in athenarian sea anemones.Zool. Verh.437484(2003).

5. Stefanik, D. J., Friedman, L. E. \& Finnerty, J. R. Collecting, rearing, spawning and inducing regeneration of the starlet sea anemone, Nematostella vectensis.Nat. Protoc.8,916-923(2013).

6. Genikhovieh, G. \& Technau, U. Inductionofspawninginthestarletseaanemonenematostellavectensis,invitrofertilizationofgametes,anddejellyingofzygotes. Cold Spring Harb. Protoc.4,pdb.prot5281(2009).

7. Notredame, C., Higgins, D. G. \& Heringa, J. T-coffee: A novel method for fast and accurate multiple sequence alignment.J. Mol. Biol.302,205-217(2000).

8. Mistry, J., Finn, R. D., Eddy, S. R., Bateman, A. \& Punta, M. Challenges in homology search: HMMER3 and convergent evolution of coiled-coil regions.Nucleic Acids Res.41,(2013).

9. Bateman, A. et al. UniProt: The universal protein knowledgebase.Nucleic Acids Res.45,D158-D169(2017).

10. Shen, W., Le, S., Li, Y. \& Hu, F. A cross-platform and ultrafast toolkit for FASTA/Q file manipulation.PLoS One(2016)doi:10.1371/journal.pone.0163962.

11. Simão, F. A., Waterhouse, R. M., loannidis, P., Kriventseva, E. V. \& Zdobnov, E. M. BUSCO: Assessing genome assembly and annotation completeness with single-copy orthologs.Bioinformatics31,3210-3212(2015).

12. Kayal, E. et al. Phylogenomics provides a robust topology of the major cnidarian lineages and insights on the origins of key organismal traits.BMC Evol. Biol.18,(2018).

13. Nosenko, T. et al. Deep metazoan phylogeny: When different genes tell different stories.Mol. Phylogenet. Evol. (2013)doi:10.1016/j.ympev.2013.01.010.

14. Nakano, H. What is Xenoturbella?Zool. Lett.(2015)doi:10.1186/s40851-015-0018-z.

15. Ying, H. et al. Comparative genomics reveals the distinct evolutionary trajectories of the robust and complex coral lineages.Genome Biology(2018)doi:10.1186/s13059-018-1552-8.

16. Edgar, R. C. MUSCLE:Multiple sequence alignment with high accuracy and high throughput.Nucleic Acids Res.32,17921797(2004).

17. Minh, B. Q. et al. IQ-TREE 2: New Models and Efficient Methods for Phylogenetic Inference in the Genomic Era.Mol. Biol. Evol.37,1530-1534(2020).

18. Whelan, S. \& Goldman, N. A general empirical model of protein evolution derived from multiple protein families using a maximum-likelihood approach.Mol. Biol. Evol.(2001)doi:10.1093/oxfordjournals.molbev.a003851. 
19. Soubrier, J. et al. The influence of rate heterogeneity among sites on the time dependence of molecular rates.Mol. Biol. Evol. (2012)doi:10.1093/molbev/mss140.

20. Hoang, D. T., Chernomor, O., Von Haeseler, A., Minh, B. Q. \& Vinh, L. S. UFBoot2: Improving the ultrafast bootstrap approximation.Mol. Biol. Evol.35,518-522(2018).

21. Guindon, S. et al. New algorithms and methods to estimate maximum-likelihood phylogenies: Assessing the performance of PhyML 3.0.Syst. Biol.59,307-321(2010).

22. Stöver, B. C. \& Müller, K. F. TreeGraph 2: Combining and visualizing evidence from different phylogenetic analyses.BMC Bioinformatics(2010)doi:10.1186/1471-2105-11-7.

23. Shimodaira, H. An approximately unbiased test of phylogenetic tree selection.Syst. Biol. (2002)doi:10.1080/10635150290069913.

24. Strimmer, K. \& Rambaut, A. Inferringconfidencesetsofpossiblymisspecifiedgenetrees.Proc. R. Soc. B Biol. Sci. (2002)doi:10.1098/rspb.2001.1862.

25. Revell, L. J. phytools: An R package for phylogenetic comparative biology (and other things).Methods Ecol. Evol. (2012)doi:10.1111/j.2041-210X.2011.00169.x.

26. Yu, G., Smith, D. K., Zhu, H., Guan, Y. \& Lam, T. T. Y. Ggtree: an R Package for Visualization and Annotation of Phylogenetic Trees With Their Covariates and Other Associated Data.Methods Ecol. Evol.8,28-36(2017).

27. Babonis, L. S., Martindale, M. Q. \& Ryan, J. F. Do novel genes drive morphological novelty? An investigation of the nematosomes in the sea anemone Nematostella vectensis.BMC Evol. Biol.(2016)doi:10.1186/s12862-016-0683-3.

28. Helm, R. R., Siebert, S., Tulin, S., Smith, J. \& Dunn, C. W. Characterization of differential transcript abundance through time during Nematostella vectensis development.BMC Genomics(2013)doi:10.1186/1471-2164-14-266.

29. Warner, J. F. et al. NvERTx: A gene expression database to compare embryogenesis and regeneration in the sea anemone Nematostella vectensis.Dev.(2018)doi:10.1242/dev.162867.

30. Pedersen, J. E., Bergqvist, C. A. \& Larhammar, D. Evolution of vertebrate nicotinic acetylcholine receptors.BMC Evol. Biol. (2019)doi:10.1186/s12862-018-1341-8.

31. Dale, R. P. et al. Identification of ion channel genes in the Acyrthosiphon pisum genome.Insect Mol. Biol.19,141-153(2010).

32. Dunn, C. W., Leys, S. P. \& Haddock, S. H. D. The hidden biology of sponges and ctenophores.Trends in Ecology and Evolution(2015)doi:10.1016/j.tree.2015.03.003.

33. Liebeskind, B. J., Hillis, D. M. \& Zakon, H. H. Convergenceofionchannelgenomecontentinearlyanimalevolution.Proc. Natl. Acad. Sci. U. S. A.(2015)doi:10.1073/pnas.1501195112.

34. Pick, K. S. et al. Improved phylogenomic taxon sampling noticeably affects nonbilaterian relationships.Mol. Biol. Evol. (2010)doi:10.1093/molbev/msq089.

35. Whelan, N. V., Kocot, K. M., Moroz, L. L. \& Halanych, K. M. Error,signal,andtheplacementofCtenophorasistertoallotheranimals.Proc. Natl. Acad. Sci. U. S. A. (2015)doi:10.1073/pnas.1503453112.

36. Simion, P. et al. A Large and Consistent Phylogenomic Dataset Supports Sponges as the Sister Group to All Other Animals.Curr. Biol.(2017)doi:10.1016/j.cub.2017.02.031.

37. Dunn, C. W. et al. Broad phylogenomic sampling improves resolution of the animal tree of life.Nature452,745-749(2008).

38. Ryan, J. F., Pang, K., Mullikin, J. C., Martindale, M. Q. \& Baxevanis, A. D. The homeodomain complement of the ctenophore Mnemiopsis leidyi suggests that Ctenophora and Porifera diverged prior to the ParaHoxozoa.Evodevo(2010)doi:10.1186/2041-9139-1-9.

39. Anctil, M., Poulain, I. \& Pelletier, C. Nitric oxide modulates peristaltic muscle activity associated with fluid circulation in the sea pansy Renilla koellikeri.J. Exp. Biol.(2005)doi:10.1242/jeb.01607.

40. Shimizu, H., Koizumi, O. \& Fujisawa, T. Three digestive movements in Hydra regulated by the diffuse nerve net in the body column.J. Comp. Physiol. A Neuroethol. Sensory, Neural, Behav. Physiol.(2004)doi:10.1007/s00359-004-0518-3.

41. Kushida, Y., Higashiji, T. \& Reimer, J. D. First observation of mole-like burrowing behavior observed in a sea pen.Mar. Biodivers. (2020)doi:10.1007/s12526-020-01054-y. 
42. 42. Revell, L. J. phytools: An R package for phylogenetic comparative biology (and other things). Methods Ecol. Evol. (2012) doi:10.1111/j.2041-210X.2011.00169.x.

43. 43. Yu, G., Smith, D. K., Zhu, H., Guan, Y. \& Lam, T. T. Y. Ggtree: an R Package for Visualization and Annotation of Phylogenetic Trees With Their Covariates and Other Associated Data. Methods Ecol. Evol. 8, 28-36 (2017).

44. 44. Babonis, L. S., Martindale, M. Q. \& Ryan, J. F. Do novel genes drive morphological novelty? An investigation of the nematosomes in the sea anemone Nematostella vectensis. BMC Evol. Biol. (2016) doi:10.1186/s12862-016-0683-3.

45. 45. Helm, R. R., Siebert, S., Tulin, S., Smith, J. \& Dunn, C. W. Characterization of differential transcript abundance through time during Nematostella vectensis development. BMC Genomics (2013) doi:10.1186/1471-2164-14-266.

46. 46. Warner, J. F. et al. NvERTx: A gene expression database to compare embryogenesis and regeneration in the sea anemone Nematostella vectensis. Dev. (2018) doi:10.1242/dev.162867.

47. 47. Pedersen, J. E., Bergqvist, C. A. \& Larhammar, D. Evolution of vertebrate nicotinic acetylcholine receptors. BMC Evol. Biol. (2019) doi:10.1186/s12862-018-1341-8.

48. 48. Dale, R. P. et al. Identification of ion channel genes in the Acyrthosiphon pisum genome. Insect Mol. Biol. 19, 141-153 (2010).

49. 49. Dunn, C. W., Leys, S. P. \& Haddock, S. H. D. The hidden biology of sponges and ctenophores. Trends in Ecology and Evolution (2015) doi:10.1016/j.tree.2015.03.003.

50. 50. Liebeskind, B. J., Hillis, D. M. \& Zakon, H. H. Convergence of ion channel genome content in early animal evolution. Proc. Natl. Acad. Sci. U. S. A. (2015) doi:10.1073/pnas.1501195112.

51. 51. Pick, K. S. et al. Improved phylogenomic taxon sampling noticeably affects nonbilaterian relationships. Mol. Biol. Evol. (2010) doi:10.1093/molbev/msq089.

52. 52. Whelan, N. V., Kocot, K. M., Moroz, L. L. \& Halanych, K. M. Error, signal, and the placement of Ctenophora sister to all other animals. Proc. Natl. Acad. Sci. U. S. A. (2015) doi:10.1073/pnas.1503453112.

53. 53. Simion, P. et al. A Large and Consistent Phylogenomic Dataset Supports Sponges as the Sister Group to All Other Animals. Curr. Biol. (2017) doi:10.1016/j.cub.2017.02.031.

54. 54. Dunn, C. W. et al. Broad phylogenomic sampling improves resolution of the animal tree of life. Nature 452, 745-749 (2008).

55. 55. Ryan, J. F., Pang, K., Mullikin, J. C., Martindale, M. Q. \& Baxevanis, A. D. The homeodomain complement of the ctenophore Mnemiopsis leidyi suggests that Ctenophora and Porifera diverged prior to the ParaHoxozoa. Evodevo (2010) doi:10.1186/2041-9139-1-9.

56. 56. Anctil, M., Poulain, I. \& Pelletier, C. Nitric oxide modulates peristaltic muscle activity associated with fluid circulation in the sea pansy Renilla koellikeri. J. Exp. Biol. (2005) doi:10.1242/jeb.01607.

57. 57. Shimizu, H., Koizumi, O. \& Fujisawa, T. Three digestive movements in Hydra regulated by the diffuse nerve net in the body column. J. Comp. Physiol. A Neuroethol. Sensory, Neural, Behav. Physiol. (2004) doi:10.1007/s00359-004-0518-3.

58. 58. Kushida, Y., Higashiji, T. \& Reimer, J. D. First observation of mole-like burrowing behavior observed in a sea pen. Mar. Biodivers. (2020) doi:10.1007/s12526-020-01054-y.

\section{Supplemental Materials}

Supplementary Table S1. Seed alignment characterizing nicotinic acetylcholine and 5HT3 receptor sequences.

Supplementary Table S2. Proteome accessions from which receptor homologs were gathered.

Supplementary Table S3. nAChRs gene homologs identified in the $N$. vectensis proteome.

Supplementary Table S4. Comparison of amino acid residues within the agonist/antagonist binding loops between Nematostella vectensis and human nAChRs.

Supplementary Table S5. Final nAChRs alignment used for phylogenetic analysis.

Page 10/16 
Supplementary Table S6. Alternative topology test results, which assess confidence in the phylogenetic placement of the Cnidariagroup

Supplementary Table S7. Residues found sites known to be critical to ACh binding in the closest related genes to mAChRs in Hydra vulgaris and Nematostella vectensis.

Supplementary Video 1. Slow peristaltic contraction in Nematostella vectensis polyps over 5 minutes in 12 ppt ASW, sped up 10fold.

Supplementary Video 2. Increased peristaltic contractions in Nematostella vectensis polyps over 5 minutes in 12 ppt ASW plus $185 \mu \mathrm{M}$ nicotine, sped up 10-fold.

Supplementary Figure $\mathbf{S 1}$. Speed of contractions in Nematostella vectensis.

Supplementary Figure S2. Linear regression of the frequency of peristaltic waves vs. nicotine concentration in Nematostella vectensis.

Supplementary Figure S3. Multiple sequence alignment illustrating that characteristic structural features found in mammalian nAChRs are also found in the Nematostella vectensis nAChRs.

Supplementary Figure S4. Comparison of the predicted number of nAChR subunits and the completeness of each genome annotation.

Supplementary Figure S5. Full phylogenetic tree for nAChRs in cnidarians and bilaterians.

Supplementary Figure S6. Phylogenetic relationships of muscarinic receptors, related rhodopsin-type GPCRs, and the closest related sequences to muscarinic receptors in cnidarians Nematostella vectensis and Hydra vulgaris.

\section{Figures}


a
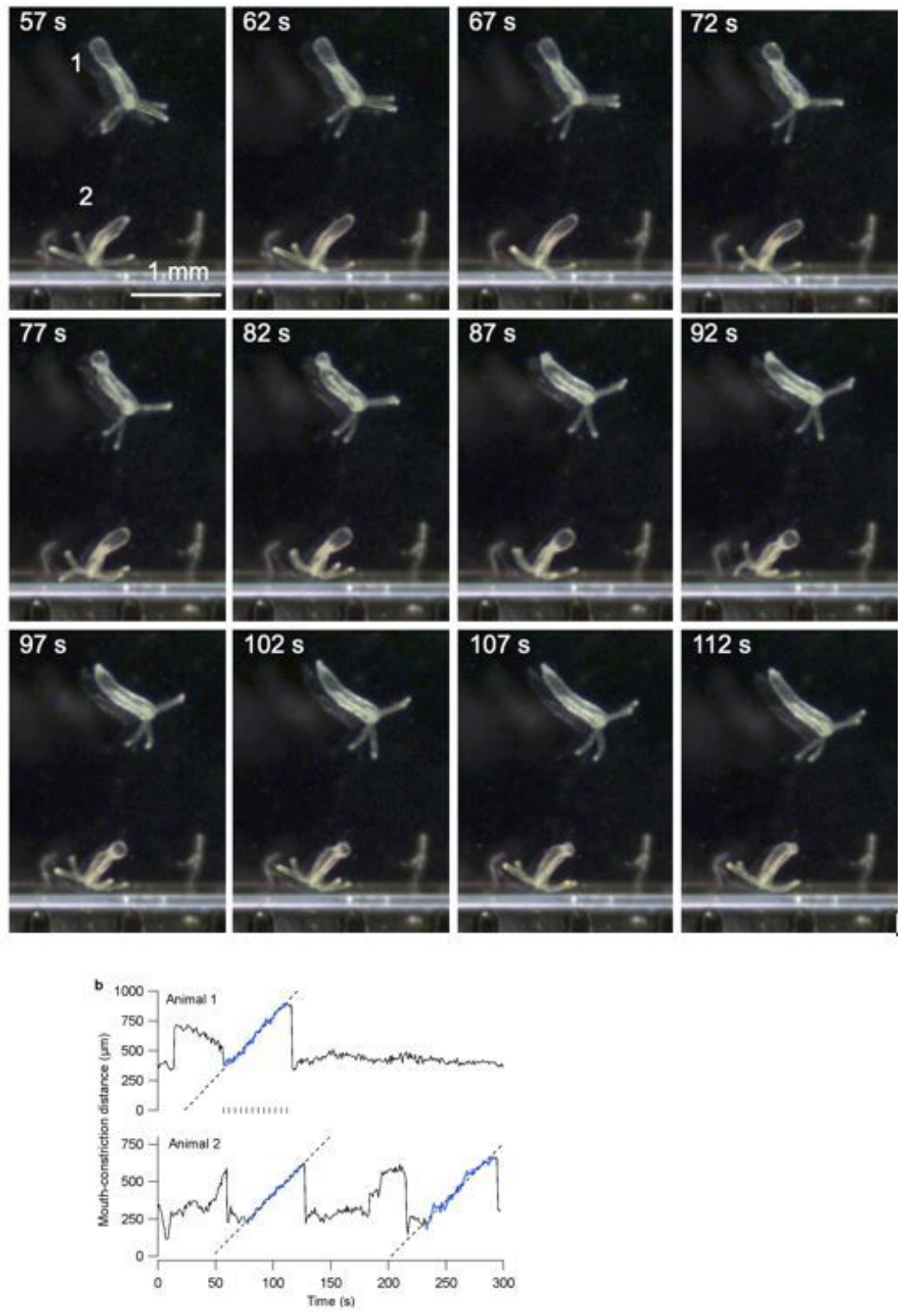

\section{Figure 1}

N. vectensis polyps undergo slow peristaltic contractions. a. A series of frames ( $5 \mathrm{~s}$ apart) from a video showing two polyps undergoing peristalsis in $12 \mathrm{ppt}$ ASW. The entire contraction of animal 1 is shown. The contraction of animal 2 began near $77 \mathrm{~s}$ and continued until $124 \mathrm{~s}$ (not shown). b. The distance between the mouth and the constricted region of the animals shown in part A. Positions were measured every 20 frames $(0.88 \mathrm{~s}$ ) throughout the $5 \mathrm{~min}$ video (black traces). The sections shown in blue indicate times when the animal was undergoing a contraction. Linear regression of these sections was used to obtain the slope which is the contraction speed. For animal 1, the contraction speed was $10.2 \mu \mathrm{m} / \mathrm{s}$; for animal 2 the speeds were 7.9 and 7.7 $\mu \mathrm{m} / \mathrm{s}$. During other parts of the record, the animal was either not contracting, was contracting while bending, or was not orientated horizontally. 



Figure 2

Distributions of wave frequency show that $\mathrm{N}$. vectensis polyps have bimodal activity levels under normal conditions, but only high activity levels with nicotine treatment. a. Histogram of the number of waves per 5 min for 808 animals in 12 ppt ASW (control). The distribution is best fit by the sum of two weighted Gaussian functions (see text). b. Histogram of the number of waves per 5 min for 187 animals exposed to $185 \mu \mathrm{M}$ nicotine. c. The distributions from A and B shown as violin plots (see Methods for details).

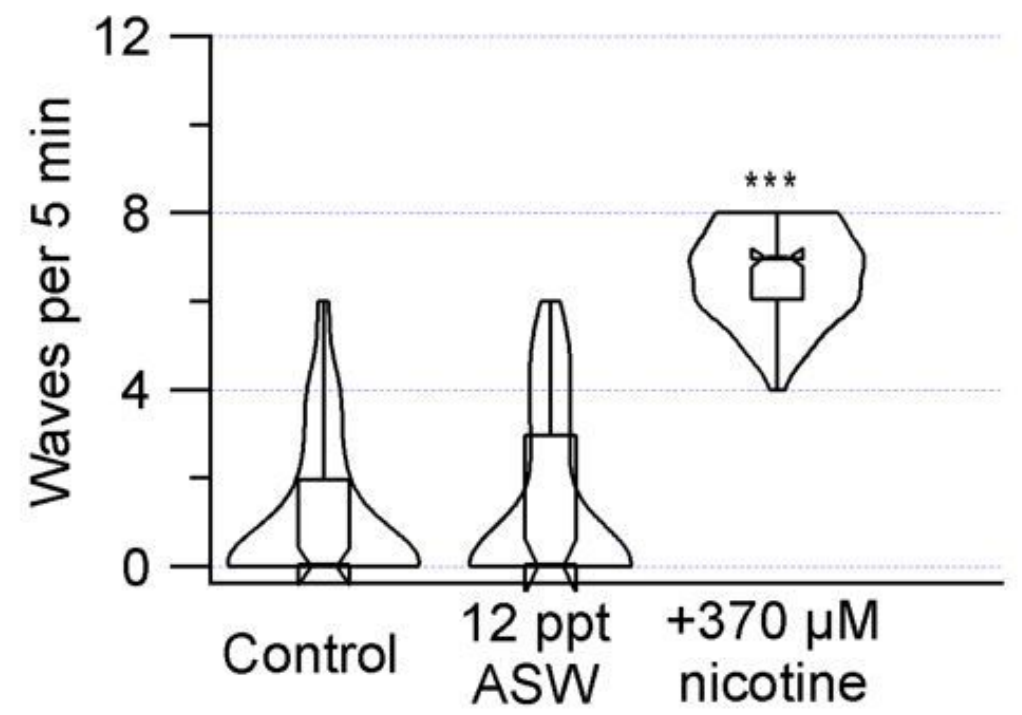

Figure 3

Addition of solution to the well does not stimulate contractions in polyps. Violin plots showing the results from 56 animals. The animals were initially in $12 \mathrm{ppt}$ ASW (control, mean frequency $=1.04$ waves per $5 \mathrm{~min}$ ). $0.01 \mathrm{ml}$ of $12 \mathrm{ppt}$ ASW was added to the well. Five minutes after the addition, the frequency of waves was the same as in control (mean frequency $=1.27$ waves per 5 min, Dunn's multiple comparison after Friedman test, $p>0.05$ ). Addition of $370 \mu \mathrm{M}$ nicotine to the well increased the frequency of waves (mean frequency $=6.63$ waves per 5 min, Dunn's multiple comparison after Friedman test, $p<0.0001$ ). 


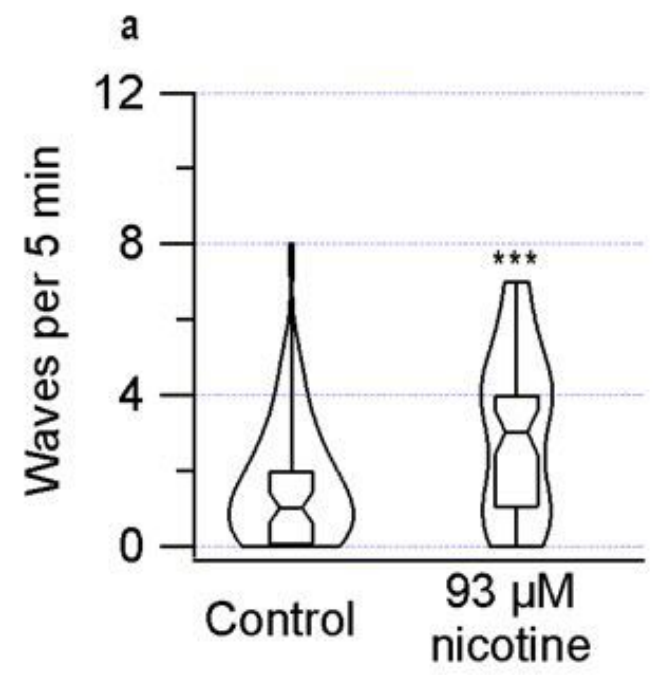

b

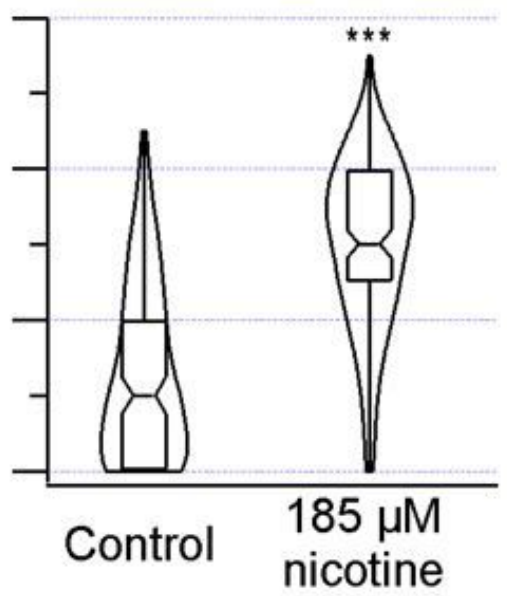

C



Figure 4

The frequency of waves increases with increasing concentrations of nicotine. $* \star \star$ indicates difference with Wilcoxon signed rank test $p<0.0001$ a. $93 \mu \mathrm{M}$ nicotine (63 animals, mean frequency $=1.64$ waves per 5 min for control, mean frequency $=2.98$ waves per $5 \mathrm{~min}$ for nicotine). b. $185 \mu \mathrm{M}$ nicotine ( 169 animals, mean frequency $=2.48$ waves per $5 \mathrm{~min}$ for control, mean frequency $=$ 6.02 waves per $5 \mathrm{~min}$ for nicotine). c. $370 \mu \mathrm{M}$ nicotine ( 55 animals, mean frequency $=1.53$ waves per 5 min for control, mean frequency $=6.62$ waves per $5 \mathrm{~min}$ for nicotine).
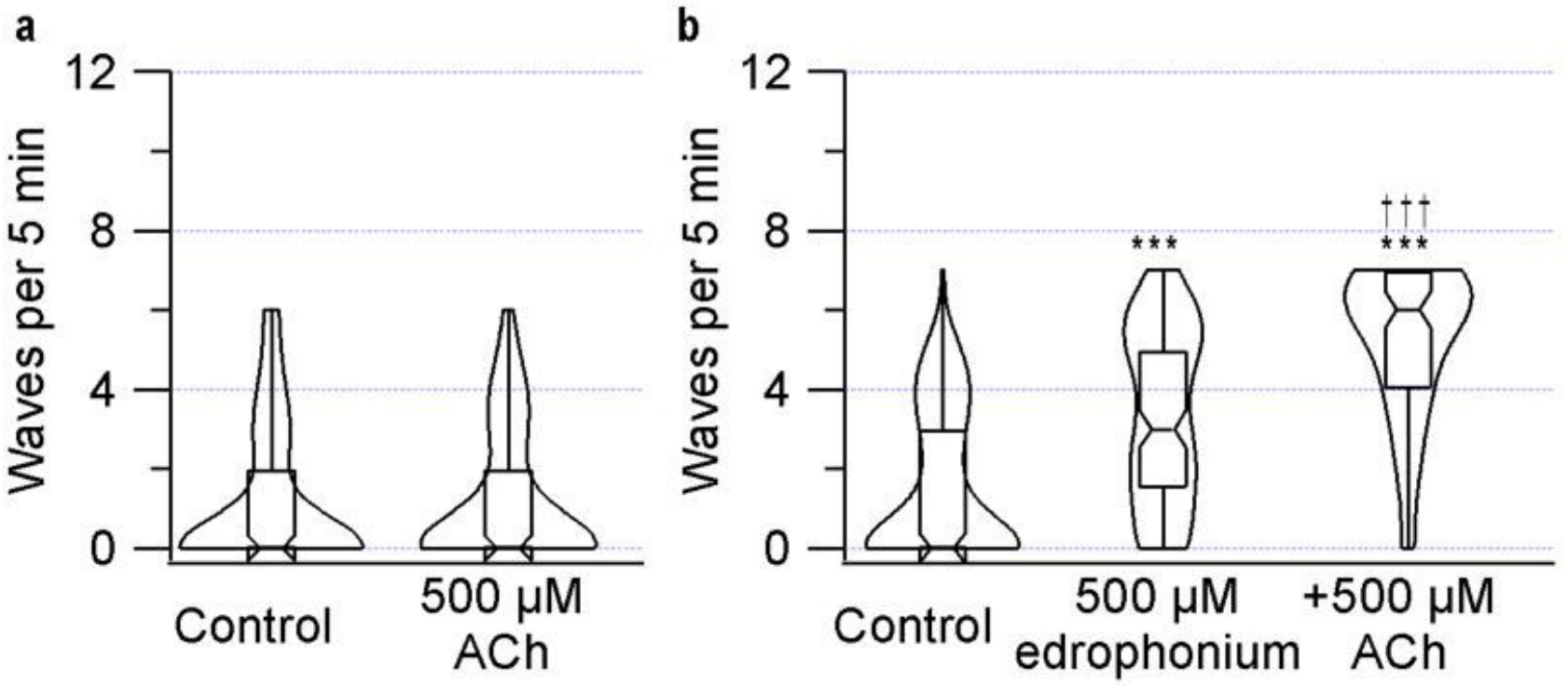

Figure 5

Edrophonium increases the effectiveness of both endogenous and exogenously applied ACh. a. Addition of $500 \mu \mathrm{M}$ ACh did not change the frequency of peristaltic waves (100 animals, mean frequency $=1.07$ waves per 5 min for control, 1.17 waves per 5 min for ACh, $\mathrm{p}>0.05)$. b. Addition of $500 \mu \mathrm{M}$ edrophonium increases the frequency of waves and addition of $500 \mu \mathrm{M}$ ACh increases 
the frequency further. (153 animals, mean frequency $=1.50$ waves per 5 min for control, 3.32 waves per 5 min for $500 \mu \mathrm{M}$ edrophonium, 5.21 waves per $5 \mathrm{~min}$ for $500 \mu \mathrm{M}$ edrophonium $+500 \mu \mathrm{M} \mathrm{ACh})$. Dunn's multiple comparison after a Friedman test shows $\mathrm{p}<0.001$ for control vs. $500 \mu \mathrm{M}$ edrophonium (***), $\mathrm{p}<0.001$ for control vs. $500 \mu \mathrm{M}$ edrophonium $+500 \mu \mathrm{M}$ ACh (***), and $\mathrm{p}<0.001$ for $500 \mu \mathrm{M}$ edrophonium vs. $500 \mu \mathrm{M}$ edrophonium $+500 \mu \mathrm{M}$ ACh (†††).

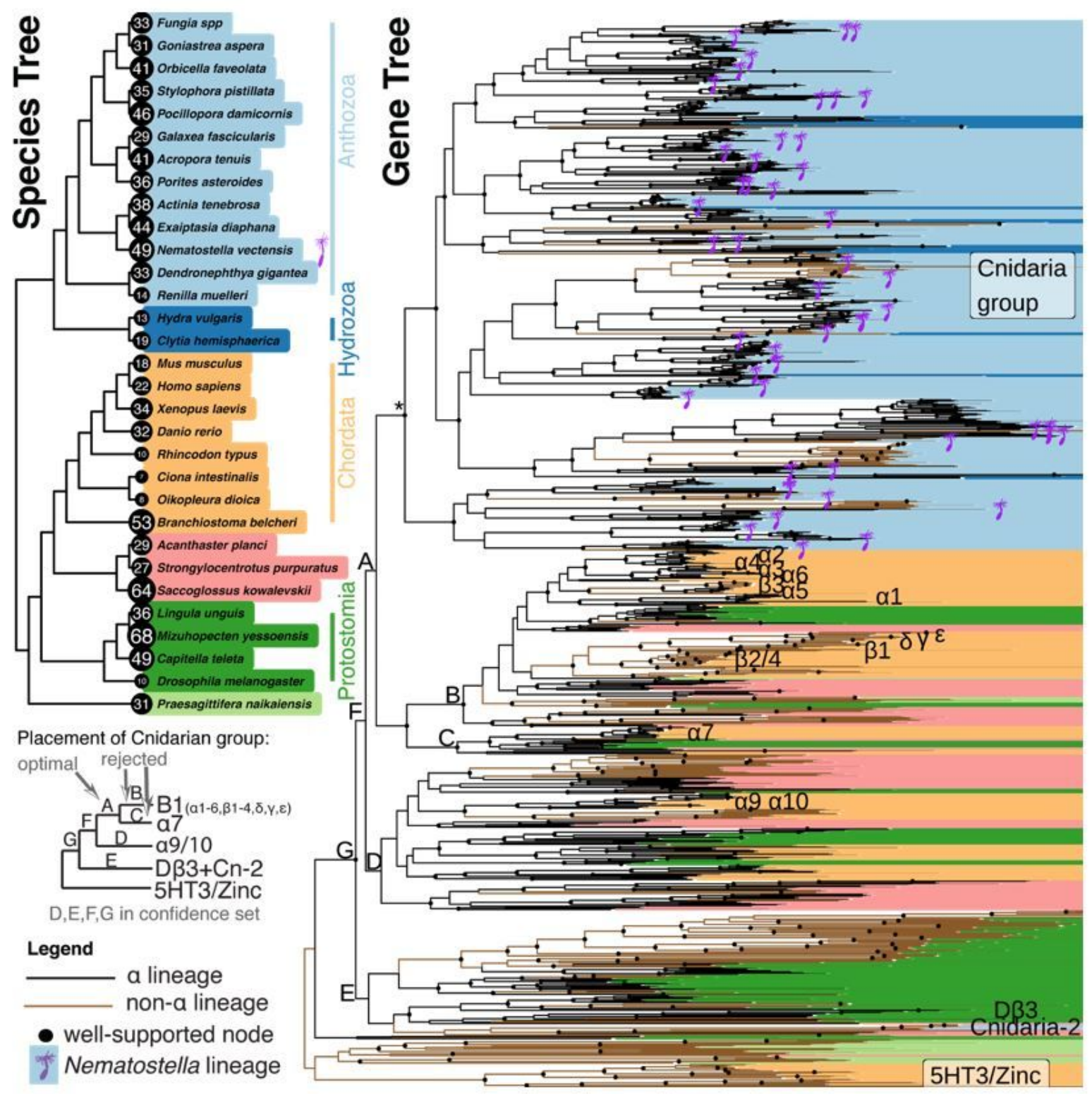

\section{Figure 6}

Phylogenetic relationships of nAChR genes in select cnidarian and bilaterian genomes shows parallel expansion. Species Tree (top left) illustrates included species with literature relationships (some nodes are unsettled; see Methods). Species names are colored by class, phylum, or superphylum. Number of nAChR gene copies identified in each genome are indicated for each tip. The protein-based Gene Tree (right) for these copies includes genes identified with a nAChR-specific hidden Markov model, with relationships calculated via maximum likelihood (see Methods). The tree is rooted with 5HT3/Zinc receptors. Almost all cnidarian receptors (light and dark blue tip labels) are part of a single, large lineage ("Cnidaria-group", indicated by *) that is sister to a large linage of bilaterian receptors (branches B and C). An additional group of cnidarian orthologs ("Cnidaria-2") is found in the D 33 group (branch E). Lineages that encode a-like subunits are colored black, while non-a lineages are colored tan. Strong ultra-fast bootstrap (>95\%) and SH-aLRT (>80\%) support is indicated by black dots at nodes. The gene tree with labels is provided as Supplementary Figure S5. Confidence in the Placement of the Cnidarian group (bottom left) was tested using ELW and AU 
topology tests (see Methods). Letters in the summary diagram correspond to branches in the gene tree. In the optimal tree, the Cnidaria-group attaches to branch A; both tests reject attachment of the Cnidaria-group to branches B and C (Supplementary Table S6). However, attachment to branches D,E,F, or G cannot be rejected from the 0.95 confidence set.

\section{Supplementary Files}

This is a list of supplementary files associated with this preprint. Click to download.

- FigueiredoVideo1.mp4

- FigueiredoVideo2.mp4

- FigueiredoSupplementalFigures1.pdf

- FigueiredoSupplementalTables.xIsx 